\title{
Fighting against kidney diseases with small interfering RNA: opportunities and challenges
}

\author{
Cheng Yang ${ }^{1,2}$, Chao Zhang ${ }^{1,2}$, Zitong Zhao ${ }^{1,2}$, Tongyu Zhu ${ }^{1,2^{*}}$ and Bin Yang $3,4,5^{*}$
}

\begin{abstract}
The significant improvements in siRNA therapy have been achieved, which have great potential applications in humans. The kidney is a comparatively easy target organ of siRNA therapy due to its unique structural and functional characteristics. Here, we reviewed recent achievements in siRNA design, delivery and application with focuses on kidney diseases, in particular kidney transplant-related injuries. In addition, the strategy for increasing serum stability and immune tolerance of siRNA was also discussed. At last, the future challenges of siRNA therapy including organ/tissue/cell-specific delivery and time-controlled silence, as well as selecting therapeutic targets, were addressed as well.
\end{abstract}

Keywords: Small interfering RNA, Kidney disease, Delivery, Off-target effect and compensative response

\section{Introduction}

RNA interference (RNAi) is a highly conserved biological phenomenon in all eukaryotes, including renal cells. In the late 1990s, due to the development of molecular biology and genetics, the biological understanding of RNA evolved from simply an intermediate between DNA and protein to a dynamic and versatile regulator that functions in genes and cells in all living organisms. In 1998, as a milestone, Fire et al. injected a few molecules of doublestranded RNA (dsRNA) into Caenorhabditis elegans and found that dsRNAs could specifically interfere with the protein expression of an endogenous gene [1]. This molecule was named small interfering RNA (siRNA) that mediates RNAi [2-4]. siRNA is able to recognize and degrade a homologous host mRNA. Therefore, the gene from which the mRNA is transcribed is silenced, which is referred to as post-transcriptional gene silencing $[5,6]$.

Although RNAi naturally exists, synthetic artificial siRNA exerts similar effects as natural endogenous microRNA (miRNA). Both sense and antisense strands of siRNA can be synthesized separately and annealed to form double stranded siRNA duplexes in vitro. After the siRNA is delivered into the cytoplasm, the artificial siRNA

\footnotetext{
*Correspondence: tyzhu_dr@163.com; by5@le.ac.uk

${ }^{1}$ Department of Urology, Zhongshan Hospital, Fudan University, Shanghai,

China

${ }^{3}$ Transplant Group, Department of Infection, Immunity and Inflammation, University Hospitals of Leicester, University of Leicester, Leicester, UK Full list of author information is available at the end of the article
}

silences the target gene using similar biological processes as endogenous miRNA. Since the introduction of 21nucleotide artificial siRNAs that triggered gene silencing in mammalian cells [7], synthetic siRNA has generated much interest in biomedical research, in which the kidney is one of important key players. siRNA as a strategic molecule has been highly expected in the field of innovative therapy. Because siRNA is highly efficient at gene silencing, it is possible to develop specific siRNA-based drugs that could target any genes, including those that have no known pharmacological antagonists or inhibitors. Different types of synthetic siRNA have been tested for their efficacy in various disease models, including cancer $[8,9]$, autoimmune disorders [10], cardiovascular injuries [11,12], and organ transplantation $[13,14]$, including native and transplanted kidney injuries [15].

As siRNA is a posttranscriptional regulator, it must first be absorbed into the target cells. Therefore, the kidney could be an excellent target organ for siRNA therapy because it benefits from rapid, vast blood flow physically and subsequent glomerular filtration and tubular absorption. In fact, systemic administration of siRNA leads to rapid uptake by the kidney, yielding a significant decrease of target protein expression [15]. Consequently, RNAi by siRNA has advantages for the treatment of renal diseases due to the unique urological system. In addition, the preservation of donor kidneys before transplantation also provides a suitable time window for the 
intervention of siRNA. Therefore, we performed a series of experiments using naked caspase- 3 siRNA to investigate its efficacy, off-target effects and compensative responses in in vitro, ex vivo and in vivo models of transplant-related renal injuries.

In this review, we highlighted the design and delivery of siRNA, its therapeutic effects, off-target responses and systematic compensations, as well as potential challenges, with a focus on kidney diseases, including ongoing clinical trials.

\section{Current principle of siRNA design}

The design of potent siRNAs has been greatly improved over the past decade. The basic criteria for choosing siRNAs includes the consideration of thermodynamic stability, internal repeats, immunostimulatory motifs, such as GC content, secondary structure, base preference at specific positions in the sense strand, and appropriate length [16].

Chemical modifications significantly enhance the stability and uptake of naked siRNAs. Importantly, siRNAs can be directly modified without crippling the silencing ability. Chemical modifications have been rigorously investigated for virtually every part of siRNA molecules, from the termini and backbone to the sugars and bases, with the goal of engineering siRNA to prolong half-life and increase cellular uptake. The most common chemical modification involves modifying the sugar moiety. For example, the incorporation of 2'-fluoro (2'-F), -O methyl, -halogen, -amine, or -deoxy can significantly increase the stability of siRNA in serum. Locked nucleic acid (LNA) has been also applied to modify siRNA. The commonly used LNA contains a methylene bridge connecting the 2'-oxygen with the 4'-carbon of the ribose ring. This bridge locks the ribose ring in the 3'-endo conformation characteristic of RNA [17]. Additionally, recent studies, including ours [18], have proven the efficacy of LNA modified siRNA in terms of prolonged half-life in serum, but without detectable adverse effects, suggesting that the natural RNAi machinery could accommodate a certain degree of alterations in the chemical structure of siRNAs [19].

\section{siRNA delivery}

The biggest obstacle faced by siRNA therapies is the in vivo delivery of genetic materials. The virus-based delivery system, while efficient, may be fatally flawed due to raised safety concerns, such as inducing mutations and triggering immunogenic and inflammatory responses [20]. Therefore, extensive research had been performed to develop efficacious non-viral delivery systems, including direct chemical modification of siRNA (as described above) and/or optimization of delivery materials, such as liposome formulation, nanoparticle conjugation and antibodies that target cellular moieties [20].
To date, studies on synthetic siRNA therapy have been performed in a variety of cell culture and rodent models [21] that produced exciting results and were cost effective, but failed to faithfully mimic human diseases. Therefore, large animal models, such as porcine models, are indispensable to compensate for the limitations of rodent models due to their greater similarity to human beings. The investigations on siRNA conducted in our laboratory have reflected this trend in the field $[14,18,22]$.

\section{In vitro delivery}

Cell culture is an important model for investigating the cellular and molecular mechanisms of diseases. Lentivirus vectors and liposomes are widely applied for the transduction of siRNA into different types of cultured renal cells. For example, silencing $c c r 2$ using its siRNA delivered by a lentivirus significantly ameliorated MCP-1 induced podocyte apoptosis under diabetic conditions [23]. Similarly, degradation of FoxO1 by transfecting its siRNA via lentiviral vectors overrode the limited cell cycle and stimulated proliferation of glomerular mesangial cells [24]. In our previous study, we transfected porcine proximal tubular cells (LLC-PK1) with synthetic caspase-3 siRNA using a cationic lipid-based transfection reagent. The caspase- 3 siRNA inhibited apoptosis and inflammation in LLC-PK1 cells that were subjected to hydrogen peroxide stimulation [25].

\section{Ex vivo delivery}

In addition to in vitro delivery of siRNA, ex vivo/in vivo siRNA delivery to target organs is an indispensable step before its clinical application. If it were directly delivered into the kidneys, siRNA could obtain higher local concentrations, which would result in improved gene silencing efficacy. During kidney transplantation, ex vivo local delivery of siRNA into the donor kidney is feasible because it could be facilitated by the unique structure of the kidney and the characteristics of kidney transplantation. Recently, we utilized an ex vivo isolated porcine kidney reperfusion system to assess the efficacy of naked caspase-3 siRNA. The caspase-3 siRNA was directly infused into the renal artery (locally) and autologous blood perfusate (mimic systemic delivery) before 24-hour cold storage (CS), followed by a further reperfusion for 3 hours. The results demonstrated that the caspase- 3 siRNA improved ischemic reperfusion (IR) injury with reduced caspase- 3 expression and apoptosis, better renal oxygenation and acid-base homeostasis [22]. These promising proof-of-principle observations provide valuable guidance for further development before siRNA clinical practice.

\section{In vivo local delivery}

Based on the anatomical and physiological characteristics of the kidney, local delivery can be achieved through 
several routes: (1) renal artery: first targeting the glomeruli or tubules [26,27]; (2) renal vein: predominately targeting tubulointerstitium [28]; (3) intra urethral: administered into the renal pelvis and interstitium [29]; and (4) sub-capsular administration: achieves intraparenchymal silencing [30]. Due to the rich blood flow through the glomeruli, siRNA injection via the renal artery followed by electroporation could silence specific genes in the glomeruli, such as TGF- $\beta$, which subsequently ameliorates matrix expansion in an experimental glomerulonephritis model [26]. However, acute kidney injury (AKI), such as IR injury, is usually characterized by tubular apoptosis and inflammatory infiltration in the tubulointerstitium, which should be suitable for local siRNA delivery via any above mentioned method.

It has been revealed that an injection of a single-dose Fas siRNA through the renal vein post ischemia provided a survival advantage in a murine IR model, which was due to the anti-apoptosis and anti-inflammation effects of the Fas siRNA [28]. Unilateral ureteral obstruction (UUO) is a well-established model for tubulointerstitial fibrosis. Xia et al. injected the siRNA of heat shock protein 47 once via the ureter at the time of UUO preparation, leading to significantly reduced fibrosis-related protein expression and a remarkable alleviation of the accompanying interstitial fibrosis [29]. Sub-capsular administration is still used in some experiments due to its unique advantages, although it requires an invasive procedure and has limitations in clinical practice. Cuevas et al. reported that an infusion of DJ-1 (an antioxidant) specific siRNA into the sub-capsule silenced DJ-1 expression in the renal cortex and increased ROS production [30].

\section{In vivo systemic delivery}

Systemic delivery is a common and convenient clinical practice, although current clinical trials using siRNAs are almost directly administered to the target site, such as the nostril, eye and lung, thereby avoiding the complexity of systemic delivery [31]. The most common method of systemic siRNA delivery is a hydrodynamic intravenous injection with hydraulic pressure to assist siRNA cell entry. However, the pharmacokinetic metabolism of siRNA is more complicated during systemic delivery because siRNAs can be rapidly degraded by nucleases in the serum and cleared by the kidney and liver. To enhance the in vivo efficacy of siRNA treatment, a variety of approaches have been attempted for both siRNA itself and delivery techniques [30-36], as mentioned above and discussed later.

Due to its anatomical and physiological characteristics, the kidney is the most preferable target organ of systemic siRNA administration. siRNA access to the kidney is thought to be dependent on the filtration and reabsorption functions of the kidney. Proximal tubule cells
(PTCs) are the primary site for rapid and extensive endocytic uptake of siRNA within the kidney following glomerular filtration. In an AKI model, naked synthetic siRNA targeting p53 that was intravenously injected 4 hours after renal ischemic injury significantly reduced upregulated p53 expression and protected both the PTCs and kidneys [37]. In another study performed by Zheng et al., siRNA was systematically injected to target complement $3(\mathrm{C} 3)$ and caspase-3 in a murine renal IR injury model. The results showed that the level of serum creatinine and blood urea nitrogen was significantly decreased in the siRNA-treated mice [38]. These studies highlighted the potential feasibility of systemically delivered siRNA for the treatment of kidney diseases in future clinical practice.

\section{siRNA therapeutic efficacy}

To date, siRNA therapy has been successfully applied for acute and chronic kidney diseases, as well as renal tumors.

\section{Acute kidney injury}

AKI is a common complication of hospitalization that has a mortality rate as high as $30-50 \%[39,40]$. IR injury is the primary cause of AKI, particularly during kidney transplantation, in which the kidney is exposed to hypoxia and experiences a series of oxidative, inflammatory and apoptotic responses [41,42]. Consequently, specific siRNAs targeting critical molecules that are involved in the processes of oxidation, inflammation and apoptosis have been developed.

Caspase-3, which mediates apoptosis and inflammation, is upregulated by IR injury. Multiple pharmacological interventions against caspase-3, including enzyme inhibitors and genetic modification, have been investigated. In recent years, our group studied the delivery and efficacy of caspase- 3 siRNA in in vitro, ex vivo and in vivo porcine models. The synthetic caspase-3 siRNA was initially tested in porcine PTCs, with or without hydrogen peroxide $\left(\mathrm{H}_{2} \mathrm{O}_{2}\right)$ stimulation. Apoptotic cells and activated IL- $1 \beta$ protein expression were significantly reduced by the caspase- 3 siRNA, with improved cell viability [25]. This outcome led to siRNA application in an isolated organ perfusion system, as described above, and the efficacy of caspase-3 siRNA was further proven [22].

We then used naked caspase- 3 siRNA in a porcine kidney auto-transplant model for the first time. The left kidney was retrieved from mini pigs and was infused with University of Wisconsin solution, with or without $0.3 \mathrm{mg}$ naked caspase- 3 siRNA, via the renal artery, which was followed by renal artery and renal vein clamping for 24-hour cold storage (CS, mimicking donor kidney preservation before transportation in clinic). After right nephrectomy, the left kidney was auto-transplanted into the right nephridial pit for 48 hours without systemic siRNA 
treatment (Figure 1). The expression of caspase-3 mRNA and active caspase- 3 protein, as well as its precursor, was downregulated by siRNA in the post-CS kidney. In the siRNA preserved post-transplant kidney, however, caspase-3 precursor was further decreased while caspase3 mRNA and its activated subunits were upregulated, which resulted in increased apoptosis and inflammation. This study indicated that the naked caspase-3 siRNA was effective for cold preservation but was not effective at protecting post-transplant kidneys, which may be due to systemic compensative responses overcoming local effects. Therefore, to overcome the systemic response and prolong the therapeutic time window, we subsequently utilized a novel, serum stable caspase-3 siRNA, both locally as before and systemically via a pre-transplantation intravenous injection, and observed the animals for up to 2 weeks post-transplantation. The effectiveness of the novel caspase-3 siRNA was confirmed by downregulated caspase- 3 mRNA and protein in the post-CS and/or posttransplant kidneys, as well as reduced apoptosis and inflammation. More importantly, renal function, associated with active caspase-3, HMGB1, apoptosis, inflammation and tubulointerstitial damage, was improved by this novel, serum stable caspase-3 siRNA [18].

p53, another pivotal protein in the apoptotic pathway, has been identified as a mediator of transcriptional responses to IR injury [43-45]. Molitoris et al. revealed that intravenously injected p53 siRNA attenuated ischemic and cisplatin-induced AKI [37]. Fujino et al. also tested the efficacy of transarterial administration of siRNA that targeted p53. p53 siRNA injected into the left renal artery immediately after ischemia improved tubular injury and downregulated GSK-3 $\beta$ expression [46]. In a diabetic mouse model, p53 inhibition by siRNA also reduced ischemic AKI [47].

Silencing other important transcription factors or immunity related receptors using siRNAs have also been studied. Renal IR injury and inflammation are related to postsurgical healing and both processes can be influenced by Toll-like receptor (TLR) signals. Effective TLR9 silencing by siRNA decreases renal cell apoptosis, mitigates AKI severity, and increases the mice survival [48]. NF- $\mathrm{B}$,

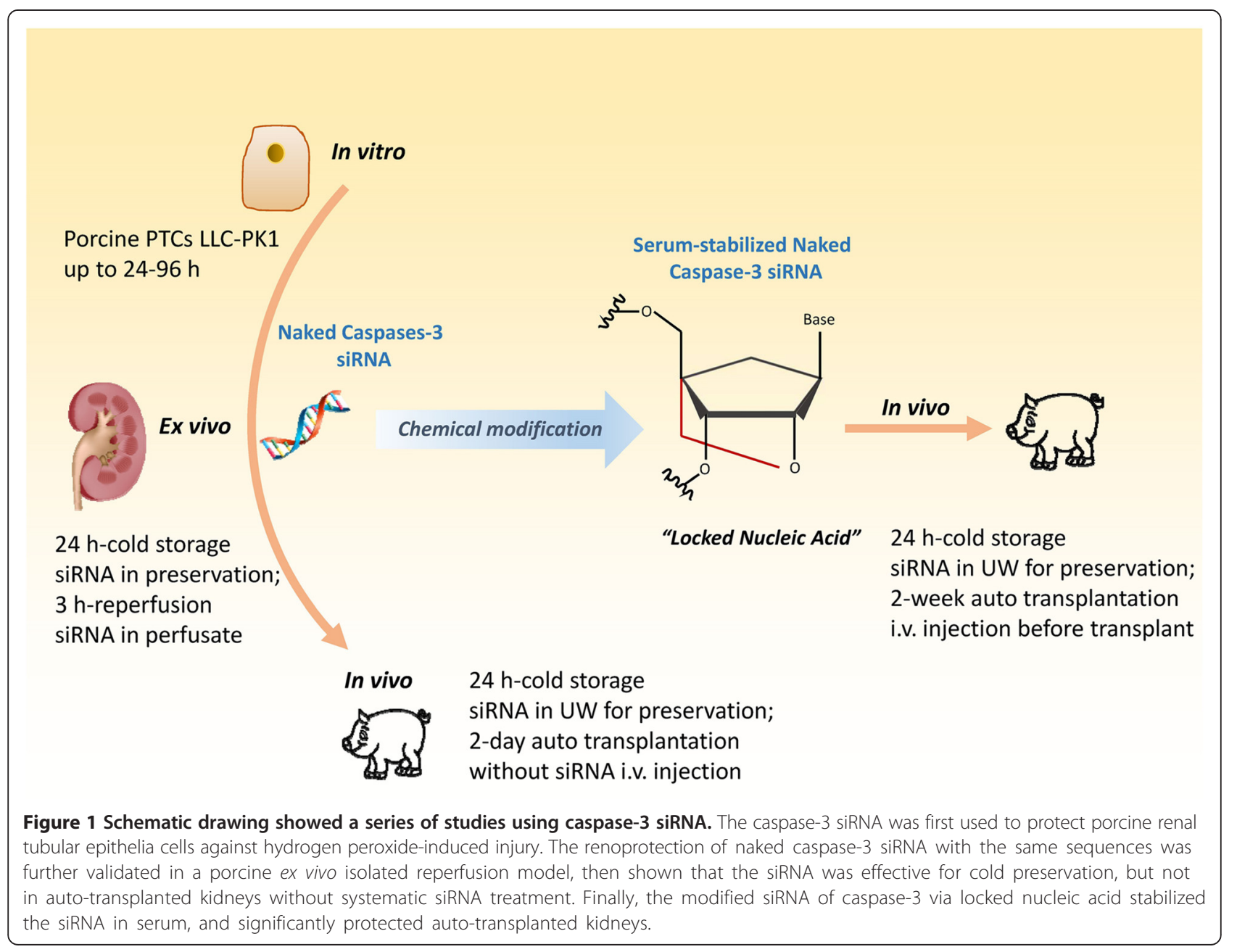


a pro-inflammatory transcription factor induced by TLR and other signals, plays a key role in AKI. NF- $\mathrm{BB}$ activation depends on the activation of the inhibitor of $\kappa B$ kinase $\beta$ (IKK $\beta)$. Wan et al. demonstrated that silencing IKK $\beta$ using siRNA diminished inflammation and protected the kidneys against IR injury [27]. These studies clearly demonstrate the therapeutic potential of siRNA-induced silencing of key AKI mediators, which are activated and involved in the pathways of apoptosis, inflammation, immunity, etc.

\section{Chronic renal disease}

Glomerulonephritis, resulting from multiple factors, is the most common primary disease leading to chronic kidney dysfunction. Interventions based on siRNA therapy for glomerulonephritis are promising, although a tissue-specific regimen has not been established. Shimizu et al. utilized the electrostatic interactions between positively charged nanocarriers, polyethylene glycol-poly (l-lysine)-polymers, with negatively charged siRNA to facilitate delivery. MAPK1 siRNA/nanocarrier complex used in a model of glomerulonephritis demonstrated that MAPK1 suppression remarkably improved kidney function, reduced proteinuria, and ameliorated glomerular sclerosis. The expression of the profibrotic marker TGF- $\beta$ was also significantly decreased after MAPK1 siRNA therapy and glomerulonephritis progression was prevented [49]. However, in contrast to AKI treatment, repeated siRNA administration will be necessary to achieve a therapeutic effect in chronic conditions. This necessity leads to some concerns for siRNA therapy, such as the medical cost and potential long-term side effects, which will be discussed later.

\section{Renal cell carcinoma}

Advanced renal cell carcinoma (RCC) is highly resistant to standard chemotherapy regimens, resulting in a 5year survival rate of only $10 \%$ for those with stage IV disease [50]. Gene therapy provides an efficient method for targeting the specific genes involved in RCC pathogenesis. For example, the HuR protein is a nucleocytoplasmic protein that plays an important role in the regulation of mRNA stability [51]. A recent study examined the effects of siRNA-mediated HuR gene silencing in multiple RCC cell lines in vitro and demonstrated a $60 \%$ reduction in tumor cell growth compared with control cells. These findings were then successfully replicated in vivo using tumor-bearing mice [52]. Shang et, al. further demonstrated the potential mechanism involved in the proliferative ability of RCC cells using siRNA technology [53]. Other siRNA experiments have revealed that histone $\mathrm{H} 3$ acetylation was responsible for preserving drug sensitivity in RCCs, which indicates the important role of epigenetics in RCC [54].
Nevertheless, silencing a single gene may cause tumor self-regulation and the development of drug resistance. Cancers, as well as other angiogenesis-related diseases, often result from the overexpression of multiple endogenous and exogenous pathogenic genes. One strategy for overcoming these challenges is using a cocktail therapy that combines multiple siRNAs targeting multiple diseasecausing genes. Indeed, siRNA cocktails have demonstrated better anti-tumor potency compared with siRNAs that target a single gene $[55,56]$ (Table 1 ).

\section{Off-target side effects/toxicities of siRNA}

Off-target effects are one of the major obstacles for siRNA therapy. The induction of various side effects may be caused by unexpected perturbations between RNAi molecules and cellular components. The off-target effects of siRNA were first reported by Jackson and colleagues in 2003 [57]. Broadly speaking, off-target effects can be siRNA specific or non-specific. The former are caused by limited siRNA complementarity to non-targeted mRNAs. The latter, resulting in immune- and toxicityrelated responses, are due to the construction of the siRNA sequence, its modification or the delivery vehicle.

The off-target effects associated with siRNA delivery fall into three broad categories: (1) microRNA-like offtarget effects, referring to siRNA-induced sequencedependent regulation of unintended transcripts through partial sequence complementarity to their 3'UTRs; (2) inflammatory responses through the activation of TLR triggered by siRNAs and/or delivery vehicles (such as cationic lipids and viruses); and (3) widespread effects on microRNA processing and function through the saturation of the endogenous RNAi machinery by exogenous siRNAs [58,59].

\section{MicroRNA-like off-target effects}

The siRNAs and microRNAs share similar machinery downstream of their initial processing. Using several different siRNAs targeting the same gene, microarray profiling showed that each siRNA produced a unique, sequence-dependent signature. Sequence analysis of offtarget transcripts revealed that the 3' UTR regions of these transcripts were complementary to the 5' end of the transfected siRNA guide strand [57]. It is now understood that for the off-targeting mechanism to occur, a perfect complementation between the nucleotide positions $2-7$ or $2-$ 8 (seed region) of the antisense strand and the 3'UTR of the transcript is necessary $[58,60]$. Base mismatches in the 5 ' end of an siRNA guide strand reduced silencing of the original set of off-target transcripts, but introduced a new set of off-target transcripts with 3' UTRs that were complementary to the mismatched guide strand [58].

RNAi regulation by miRNAs involves partial complementarity between the target RNA and miRNA. Because 
Table 1 Studies on kidney diseases using siRNAs

\begin{tabular}{|c|c|c|c|c|c|c|c|}
\hline Investigators & Model & Modification & Transfection & Delivery & Target gene & Pathway & Reference \\
\hline Yang et al. & Porcine LLC-PK1 & - & Cationic lipid & In vitro & Caspase-3 & Apoptosis & {$[25]$} \\
\hline Yang et al. & Porcine & - & UW solution & $\begin{array}{l}\text { Ex vivo, renal artery injection and } \\
\text { autologous blood perfusate }\end{array}$ & Caspase-3 & Apoptosis & {$[22]$} \\
\hline Yang et al. & Porcine & - & UW solution & In vivo, renal artery injection & Caspase-3 & Apoptosis & [14] \\
\hline Yang et al. & Porcine & LNA & UW solution & $\begin{array}{l}\text { In vivo, renal artery injection and } \\
\text { systemically iv. }\end{array}$ & Caspase-3 & Apoptosis & [18] \\
\hline Liu et al. & Mice & - & Plasmid & In vivo, hydrodynamic injection iv. & TLR9 & Innate immunity & {$[48]$} \\
\hline Hamar et al. & Mice & $\begin{array}{l}\text { 2'-O-ACE-RNA } \\
\text { phosphoramidites }\end{array}$ & Phosphate buffered saline & In vivo, hydrodynamic injection iv. & Fas & Apoptosis & {$[28]$} \\
\hline Wan et al. & Rats & - & Phosphate buffered saline & In vivo, renal artery injection & IKK $\beta$ & Inflammation & [27] \\
\hline Xia et al. & Mice & - & Cationized gelatin microspheres & In vivo, injected via ureter & HSP47 & collagen-producing, fibrosis & [29] \\
\hline Molitoris et al. & Rats & 2'O-methylation & Lipofectamine 2000 & In vivo, iv. & p53 & Apoptosis & [37] \\
\hline Zheng et al. & Mice & - & Lipofectamine 2000 & In vivo, hydrodynamic injection iv. & $\begin{array}{l}\text { Complement } 3 \\
\text { and caspases-3 }\end{array}$ & Complement and apoptosis & [38] \\
\hline Shimizu et al. & Mice & - & Nanocarrier complexation & In vivo, ip. & MAPK1 & Immunity & [49] \\
\hline Shang et al. & ACHN, A498 RCC cells & - & Lipofectamine 2000 & In vitro & HIF-1a, HIF-2a & Tumorigenesis & [53] \\
\hline Juengel et al. & Caki-1 RCC cells & - & Transfection reagent supplied by Qiagen & In vitro & HDAC1, HDAC2 & Acetylation & {$[54]$} \\
\hline Fujino et al. & Mice & - & Cationic lipid & In vivo, renal artery injection & p53 & Apoptosis & [46] \\
\hline
\end{tabular}


miRNAs cause gene silencing through mRNA degradation and translation inhibition, the siRNA mediated offtarget effects may also be acting at two levels. For this reason, there should be greater emphasis on improving siRNA design, as well as monitoring gene and protein levels following RNAi therapy to account for any offtarget effects.

\section{Recognition/Stimulation by the innate immune system}

The recognition and stimulation of the immune system is a nonspecific off-target effect of siRNA therapy. The RNA-sensing pattern recognition receptors (PRRs), localized in endosomes, are the most important components of the innate immune system. The responses of PRRs to siRNAs are either TLR-mediated or non-TLRmediated. The PRR responses are also associated with siRNA sequence specific side effects and have recently attracted lots of attentions from researchers [61]. RNAsensing TLRs (TLR3 and TLR7) are predominantly located intracellularly and recognize nucleic acids released from invading pathogens. The non-TLR-mediated innate immune responses triggered by siRNA binding are linked to RNA-regulated expression of protein kinase (PKR) and retinoic acid inducible gene 1 (RIG1), which further induce caspase- 3 and NF- $\mathrm{KB}$ expression, respectively. The activation of PRRs generates excessive cytokine release and subsequent inflammation [62].

Based on this second type of off-target RNAi effects, our group further investigated the mechanism of how short-acting caspase-3 siRNA impaired post-transplanted kidneys. The results suggested that the amplified inflammatory responses in caspase- 3 siRNA preserved autotransplant kidneys were associated with TLR3, TLR7 and PKR activation, which may be due to systemic compensative responses, although persistent actions initiated by short-acting caspase-3 siRNA cannot be completely excluded [63]. Other studies have also indicated that the horseshoe-like structure of TLR3 facilitates dsRNA recognition $[64,65]$. Interactions between TLR3 and dsRNA were originally reported in 2001 when TLR3-deficient mice exhibited reduced immune responses to dsRNA viruses [66].

Several studies have demonstrated that the immune response to siRNAs is cell type-dependent, due to the selective expression of TLRs. siRNAs stimulate monocytes and myeloid dendritic cells through TLR8 to produce pro-inflammatory cytokines, or activate plasmacytoid dendritic cells through TLR7 to produce type I interferons [67-69]. In addition, the volume of hydrodynamic naked siRNA delivery influences immune activation. Rácz et al. compared the immune responses induced by $50 \mu \mathrm{g}$ siRNA dissolved in either low-volume $(1 \mathrm{~mL} / \mathrm{mouse})$ or highvolume ( $10 \%$ of body weight, $2.5 \mathrm{~mL} /$ mouse in average) physiological salt solution delivered in vivo. Low-volume hydrodynamic injection induced slight alanine aminotransferase (ALT) elevation and mild hepatocyte injury, whereas high-volume hydrodynamic injection resulted in higher ALT levels and extensive hepatocyte necrosis. High-volume hydrodynamic injection also led to a timedependent slight increase in IFN-related gene expression [70]. Collectively, these studies suggest that there is a need for improving siRNA design, establishing experimental controls and carefully interpreting results.

\section{Systemic compensative actions post siRNA treatment}

Silencing the target mRNA occasionally induces systemic compensative actions in vivo. In our previous research, the unmodified naked caspases-3 siRNAs aggravated renal graft injury [14]. A potential reason for this effect is due to the kidney structure. The pore size of the glomerular filtration barrier is approximately $8 \mathrm{~nm}$ and naked siRNA has been observed to pass through this barrier into the urine [20]. Upon reperfusion, the caspases-3 siRNA in the preserved kidney was flushed, while the local siRNA in the post-CS kidneys may also rapidly disappear due to a surge of blood flow into the kidney, leading to siRNA degradation and elimination. After transplantation, a series of in vivo complementary responses to the lower level of caspase-3 mRNA in the post-CS kidneys was initiated, which first led to an increase in caspase-3 mRNA synthesis. As the consequence of siRNA degeneration and caspase- 3 mRNA synthesis, the level of caspase- 3 mRNA increased in the siRNA preserved post-transplant kidneys. It has been suggested that a post-transplant feedback loop was initiated by the effective delivery of caspase- 3 siRNA during CS, which led to increased mRNA synthesis after transplantation. However, with improved nuclease stability, the LNA modified caspases-3 siRNA protected both the post-CS and post-transplant renal grafts. More importantly, the modified siRNAs did not induce systemic compensative actions, which was proven by monitoring systemic inflammation [18].

\section{From bench to bedside: clinical trials}

The numbers of RNAi-based preclinical studies and clinical trials have grown over the past several years. To date, there have been 27 registered clinical trials using siRNA worldwide. These studies include retinal degeneration, dominantly inherited brain and skin diseases, viral infections, respiratory disorders, metabolic diseases and, of particular note, kidney diseases. In 2011, Quark Pharmaceuticals completed a phase I, randomized, double-blind, dose escalation, safety and pharmacokinetic study (NCT00554359) on QPI-1002, also designated I5NP, which was a synthetic siRNA that temporarily inhibits p53 expression that is in early development for acute kidney failure therapy. I5NP is the first siRNA to be systemically administered in humans. Based on data from animal studies, 
the intravenous injection in the human studies was performed within 4 hours of bypass surgery and pharmacokinetic data were obtained during the first 24 hours. Follow-up was conducted for safety and dose-limiting toxicities until hospital discharge and then by phone 6-12 months after surgery. Recently, Quark initiated a subsequent clinical trial to determine whether a single administration of I5NP can prevent delayed graft function in kidney transplant recipients. Data from this study will be used to identify I5NP doses for follow-on efficacy studies (NCT00802347). Another ongoing phase I trial investigating solid tumors, including RCCs, was conducted by Calando Pharmaceuticals. The investigators used CALAA01, whose active ingredient is a type of siRNA, to inhibit tumor growth and/or reduce tumor size. This siRNA inhibits the expression of the M2 subunit of ribonucleotide reductase and resists nuclease degradation by using a stabilized nanoparticle that targets tumor cells (NCT00689065, the above clinical trials can be found at ClinicalTrials.gov, Table 2).

\section{Perspectives and challenges}

Despite the enormous potential of siRNA therapy, additional research must be performed before its large-scale clinical application.

\section{Target gene selection}

Genome-wide or pathway-specific siRNA libraries have become available using high-throughput screening approaches. Establishing in vitro pre-screening leads to signaling pathway prediction and target validation in in vivo renal disease. However, choosing one or a set of reasonable target gene(s) is the key for designing specific siRNA treatments. The pathophysiological changes during kidney disease, like any other disease, refer to a complex gene and protein regulation network. For example, the network that exists during kidney transplantation involves the original conditions of the donors and the interactions between the donor kidneys and the recipients, which could direct the progression, as well as the recovery, of the injury. Fortunately, transcriptome measurements of the transplant kidney may provide a comprehensive understanding of gene regulation and would be beneficial for target gene selection.
Mueller et al. analyzed the transcriptome of postreperfusion implant biopsies in living donors (LD) and deceased donors (DD). Hundreds of mRNAs were identified that predicted delayed graft function [71]. In a recent prospective study using human post-transplant kidney biopsies, 20 mRNAs and two miRNAs were identified as molecular signatures of AKI. Elevated secretory leukocyte peptidase inhibitor in AKI allografts was validated and miR-182-5p was identified as a molecular regulator [72]. These genes could be used as potential targets of siRNA therapy. We recently identified 3 times more differentially expressed genes in renal allograft biopsies between living donors and cadaveric donors at 30 min than 3 months post-transplantation. The majority of these differentially expressed genes are responsible for acute responses at $30 \mathrm{~min}$, but involved in inflammation, nephrotoxicity and proliferation at 3 months (Figure 2). These divergent transcriptome signatures between two types of donors might be linked with not only the initial injury of the donors, but also the immune responses of the recipients.

Another method for selecting target genes is by identifying their translation product proteins. To find a single or a set of crucial protein(s) involved in kidney allograft rejection, we explored potential transcriptional factors and regulation networks in 352 kidney transplant recipients, of which 85 suffered from acute rejection (AR). The results demonstrated that the dominant processes and responses were associated with inflammation and complement activation in AR. A number of transcription factors were identified in AR patients, including NF- $\mathrm{kB}$, signal transducer and activator of transcription (STAT) 1 and STAT3 [73]. Our recent study further revealed inflammation-derived kidney allograft injury, such as $\mathrm{AR}$, chronic rejection, and impaired renal function without rejection. We identified 12 common proteins and 11 level-specific proteins from the phenotype-related protein-protein interaction networks [74]. These potential biomarkers also provide valuable targets for transplantrelated injury siRNA design.

\section{Timely application}

Compared with shRNA, an advantage of siRNA for AKI therapy is the time-controlled treatment. Silencing the

Table 2 Clinical trials of siRNA therapy in kidney diseases

\begin{tabular}{|c|c|c|c|}
\hline Study & Target/siRNA drug & Status & Disease \\
\hline NCT00554359 & I5NP & Phase I, completed & Kidney injury; Acute renal failure \\
\hline NCT00802347 & $15 N P$ & Phase $\mid / I$, active, not recruiting & Delayed graft function in kidney transplantation \\
\hline NCT00689065 & $\begin{array}{l}\text { M2 subunit of ribonucleotide } \\
\text { reductase/CALAA-01 }\end{array}$ & Phase I, terminated & Solid tumor cancers \\
\hline NCT02166255 & $\begin{array}{l}\text { siRNA-transfected peripheral } \\
\text { blood mononuclear cells APN401 }\end{array}$ & Phase I, not yet recruiting & $\begin{array}{l}\text { Melanoma, kidney cancer, pancreatic cancer, or other solid } \\
\text { tumors that are metastatic or cannot be removed by surgery }\end{array}$ \\
\hline
\end{tabular}




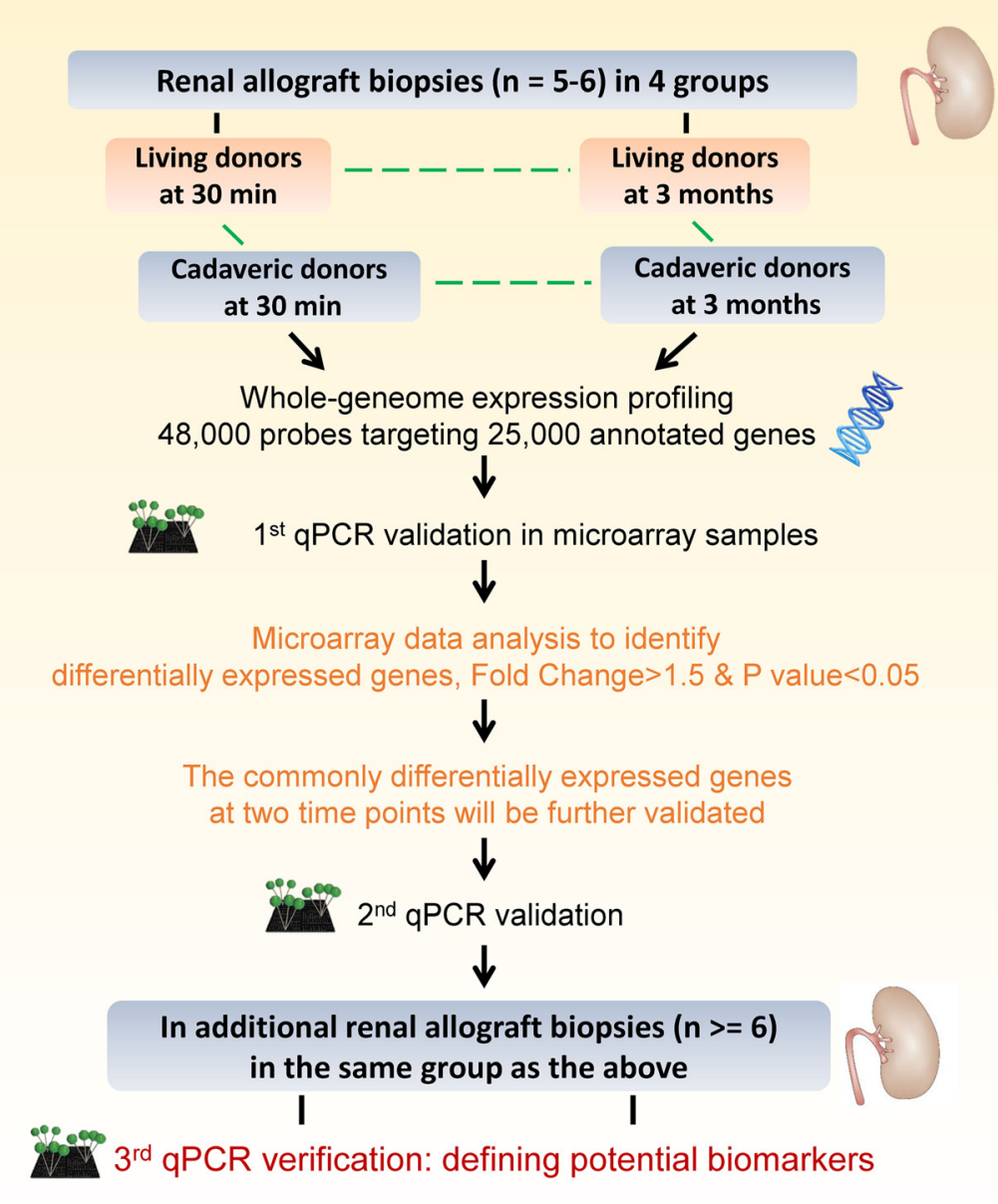

Figure 2 Study design of identifying and validating potential targets for siRNA treatment using human transplant kidney biopsies.

target gene for a short time or a long time should be addressed before RNAi application. The silenced genes may be multifunctional according to the surrounding milieu. For example, caspase-3, generally considered an executor in cell apoptosis, should be inhibited in tissue injuries. However, it is also a loyal scavenger in malignant transformation cells, which could be an unavoidable side effect in any caspase-3-targeting siRNA therapy. For AKI, siRNA ineffectiveness is needed after the therapeutic time window. Additionally, siRNA application avoids intracellular traffic. In certain circumstances, shRNA delivery could be harmful to the organ or even fatal. A study from Grimm et al. investigated the long-term effects of sustained high-level shRNA expression in the livers of adult mice. An evaluation of 49 distinct adeno-associated virus/shRNA vectors, with unique lengths and sequences that were directed against six targets, showed that 36 vectors resulted in dose-dependent liver injury, with 23 ultimately causing death. The observed morbidity was associated with the downregulation of liver-derived microRNAs (miRNAs), indicating possible competition of the latter with shRNAs (through saturation of the endogenous RNAi machinery by the exogenous siRNAs) for the limited cellular factors required for the processing of various small RNAs [75]. Therefore, controlling intracellular shRNA expression levels will be imperative, but siRNA would not influence the endogenous process of RNA degradation mediated by miRNAs.

\section{Cell-specific delivery}

For optimized RNAi therapy, it should be determined whether regional delivery with partial knockdown or systemic delivery with global knockdown is required. However, there is still a lack of target specificity during systemic siRNA delivery.

It is known that p53 in PTCs promotes AKI, whereas p53 in other tubular cells does not [40]. Therefore, the design of PTC-specific p53 siRNA is necessary. It is also expected that, for example, apoptosis-inducing siRNA should be directly delivered into tumor cells, rather than the surrounding normal cells. 
Recently, antibody conjugation technology has made tumor-targeting drug delivery systems available. In general, these systems consist of a tumor recognition moiety and a cytotoxic warhead connected directly or through a suitable linker to form a conjugate. The conjugate can be regarded as a "guided molecular missile" that specifically targets tumor unique antigens [76]. Inspired by cancer therapy strategies, siRNAs have also been "packed" to be delivered to target organs, even cells. Recently, a type of asymmetric liposome particle (ALP) has been developed, which highly efficiently encapsulates siRNA without nonspecific cell penetration. Two types of lipid inverted micelles have been prepared for the purpose of obtaining asymmetric liposome particles. The ALPs protected siRNA from ribonuclease degradation. ALPs without any surface modification elicited almost no uptake into cells, while the polyarginine peptide surfacemodified ALPs induced nonspecific cell penetration. The conjugation of an anti-human epidermal growth factor receptor antibody (anti-EGFR) to the ALPs induced EGFR-mediated uptake into non-small cell lung cancer cell lines, but not into NIH-3 T3 cells that do not have the receptor [77]. This result represents great progress in siRNA delivery system development. Antibody-mediated specific recognition may be more mainstream than virusmediated recognition in the future.

Nanoparticulate systems have emerged in last few years as an alternative material for advanced diagnostic and therapeutic applications in medicine. Compared with molecular medicine, nanotechnology offers many advantages that overcome the range of challenges and barriers summarized in the previous section, particularly the bioavailability and biodistribution of therapeutic agents. The first remarkable property of nanoparticles is their superior in vivo retention due to decreased enzymatic degradation and sequestration by phagocytes in the reticuloendothelial system. This property is primarily attributed to their immunochemically inert surfaces that are in contact with the biological environment. Methods of conjugating siRNAs with other inert and biocompatible molecules, such as cholesterol and long-chain fatty acids, have also been reported $[78,79]$.

\section{Minimizing side effects}

The knockdown of two or more genes simultaneously using an siRNA cocktail has been recently reported. Many applications of siRNA cocktails have demonstrated significant benefits compared with siRNA targeted to a single gene, particularly in anti-cancer and anti-viral therapy $[56,80]$. A high concentration of individual siRNAs may represent the key off-target effect in terms of competition for endogenous miRNA biogenesis machinery. Therefore, the other advantage of an siRNA cocktail is the relatively low concentration of each siRNA, which may reduce off-target signatures without sacrificing silencing potency [55].

\section{Conclusions}

The kidney is a comparatively easy target organ for siRNA therapy due to its unique structural and functional characteristics. siRNA intervention is effective, feasible and has great potential for fighting against kidney diseases. The safety of siRNA therapy has been proven by rapidly emerging clinical studies and off-target and compensative responses can be managed using several strategies. We believe that optimized siRNA therapy, in conjunction with advanced genetic screening technologies, could facilitate timely and specific treatment for kidney diseases, as well as other organ diseases in the near future.

\section{Competing interests}

The authors declare that they have no competing interests.

\section{Authors' contributions}

Cheng Yang and Bin Yang conceived and prepared the manuscript. Bin Yang contributed throughout the preparation and edited the manuscript. Bin Yang, Tongyu Zhu and Cheng Yang provided foundation support. Chao Zhang and Zitong Zhao revised the manuscript. All authors read and approved the final manuscript.

\section{Acknowledgements}

This study was supported by the UK-China Fellowship for Excellence, Department for Business Innovation and Skills (to BY); University Hospitals of Leicester NHS Trust, UK, and National Natural Science Foundation of China (81170689 to BY, 81270833 to TZ and 81400752 to $C Y$ ).

\section{Author details}

'Department of Urology, Zhongshan Hospital, Fudan University, Shanghai, China. ${ }^{2}$ Shanghai Key Laboratory of Organ Transplantation, Shanghai, China. ${ }^{3}$ Transplant Group, Department of Infection, Immunity and Inflammation, University Hospitals of Leicester, University of Leicester, Leicester, UK. ${ }^{4}$ Department of Nephrology, Affiliated Hospital of Nantong University, Nantong, China. ${ }^{5}$ Basic Medical Research Centre, Medical School of Nantong University, Nantong, China.

Received: 24 October 2014 Accepted: 12 January 2015

Published online: 01 February 2015

\section{References}

1. Fire A, Xu S, Montgomery MK, Kostas SA, Driver SE, Mello CC. Potent and specific genetic interference by double-stranded RNA in Caenorhabditis elegans. Nature. 1998;391:806-11.

2. Brosnan CA, Voinnet O. Cell-to-cell and long-distance siRNA movement in plants: mechanisms and biological implications. Curr Opin Plant Biol. 2011;14:580-7.

3. Pantaleo V. Plant RNA silencing in viral defence. Adv Exp Med Biol. 2011;722:39-58.

4. Dang Y, Yang Q, Xue Z, Liu Y. RNA interference in fungi: pathways, functions, and applications. Eukaryot Cell. 2011;10:1148-55.

5. Doi N, Zenno S, Ueda R, Ohki-Hamazaki H, Ui-Tei K, Saigo K. Shortinterfering-RNA-mediated gene silencing in mammalian cells requires Dicer and elF2C translation initiation factors. Curr Biol. 2003;13:41-6.

6. Hammond SM, Bernstein E, Beach D, Hannon GJ. An RNA-directed nuclease mediates post-transcriptional gene silencing in Drosophila cells. Nature. 2000:404:293-6.

7. Elbashir SM, Harborth J, Lendeckel W, Yalcin A, Weber K, Tuschl T. Duplexes of 21-nucleotide RNAs mediate RNA interference in cultured mammalian cells. Nature. 2001;411:494-8.

8. Wang Z, Rao DD, Senzer N, Nemunaitis J. RNA interference and cancer therapy. Pharm Res. 2011;28:2983-95. 
9. Petrocca F, Lieberman J. Promise and challenge of RNA interference-based therapy for cancer. J Clin Oncol. 2011;29:747-54.

10. Jeker LT, Bluestone JA. Small RNA regulators of T cell-mediated autoimmunity. J Clin Immunol. 2010;30:347-57.

11. Kowalski PS, Leus NG, Scherphof GL, Ruiters MH, Kamps JA, Molema G. Targeted siRNA delivery to diseased microvascular endothelial cells: cellular and molecular concepts. IUBMB Life. 2011;63:648-58.

12. Latronico MV, Condorelli G. RNA silencing: small RNA-mediated posttranscriptional regulation of mRNA and the implications for heart electropathophysiology. J Cardiovasc Electrophysiol. 2009;20:230-7.

13. Li F, Mahato RI. RNA interference for improving the outcome of islet transplantation. Adv Drug Deliv Rev. 2011;63:47-68.

14. Yang C, Jia Y, Zhao T, Xue Y, Zhao Z, Zhang J, et al. Naked caspase 3 small interfering RNA is effective in cold preservation but not in autotransplantation of porcine kidneys. J Surg Res. 2013;181:342-54.

15. Racz Z, Hamar P. RNA interference in research and therapy of renal diseases. Contrib Nephrol. 2008;159:78-95.

16. Chaudhary A, Srivastava S, Garg S. Development of a software tool and criteria evaluation for efficient design of small interfering RNA. Biochem Biophys Res Commun. 2011:404:313-20.

17. Elmen J, Thonberg $\mathrm{H}$, Ljungberg $\mathrm{K}$, Frieden $\mathrm{M}$, Westergaard $\mathrm{M}$, $\mathrm{Xu}$ Y, et al. Locked nucleic acid (LNA) mediated improvements in siRNA stability and functionality. Nucleic Acids Res. 2005;33:439-47.

18. Yang C, Zhao T, Zhao Z, Jia Y, Li L, Zhang Y, et al. Serum-stabilized naked caspase-3 siRNA protects autotransplant kidneys in a porcine model. Mol Ther. 2014;22:1817-28.

19. Mook OR, Baas F, de Wissel MB, Fluiter K. Evaluation of locked nucleic acid-modified small interfering RNA in vitro and in vivo. Mol Cancer Ther. 2007:6:833-43.

20. Kanasty R, Dorkin JR, Vegas A, Anderson D. Delivery materials for siRNA therapeutics. Nat Mater. 2013;12:967-77.

21. Deng Y, Wang CC, Choy KW, Du Q, Chen J, Wang Q, et al. Therapeutic potentials of gene silencing by RNA interference: principles, challenges, and new strategies. Gene. 2014;538:217-27.

22. Yang B, Hosgood SA, Nicholson ML. Naked small interfering RNA of caspase-3 in preservation solution and autologous blood perfusate protects isolated ischemic porcine kidneys. Transplantation. 2011;91:501-7.

23. Nam BY, Paeng J, Kim SH, Lee SH, Kim do H, Kang HY, et al. The MCP-1/ CCR2 axis in podocytes is involved in apoptosis induced by diabetic conditions. Apoptosis. 2012;17:1-13.

24. Liu F, Ma XJ, Wang QZ, Zhao YY, Wu LN, Qin GJ. The effect of FoxO1 on the proliferation of rat mesangial cells under high glucose conditions. Nephrol Dial Transplant. 2014;29:1879-87.

25. Yang B, Elias JE, Bloxham M, Nicholson ML. Synthetic small interfering RNA down-regulates caspase-3 and affects apoptosis, IL-1 beta, and viability of porcine proximal tubular cells. J Cell Biochem. 2011;112:1337-47.

26. Takabatake Y, Isaka Y, Mizui M, Kawachi H, Shimizu F, Ito T, et al. Exploring RNA interference as a therapeutic strategy for renal disease. Gene Ther. 2005;12:965-73.

27. Wan X, Fan L, Hu B, Yang J, Li X, Chen X, et al. Small interfering RNA targeting IKKbeta prevents renal ischemia-reperfusion injury in rats. Am J Physiol Renal Physiol. 2011;300:F857-63.

28. Hamar P, Song E, Kokeny G, Chen A, Ouyang N, Lieberman J. Small interfering RNA targeting Fas protects mice against renal ischemia-reperfusion injury. Proc Natl Acad Sci U S A. 2004;101:14883-8.

29. Xia Z, Abe K, Furusu A, Miyazaki M, Obata Y, Tabata Y, et al. Suppression of renal tubulointerstitial fibrosis by small interfering RNA targeting heat shock protein 47. Am J Nephrol. 2008;28:34-46.

30. Cuevas S, Zhang Y, Yang Y, Escano C, Asico L, Jones JE, et al. Role of renal DJ-1 in the pathogenesis of hypertension associated with increased reactive oxygen species production. Hypertension. 2012;59:446-52.

31. Gooding M, Browne LP, Quinteiro FM, Selwood DL. siRNA delivery: from lipids to cell-penetrating peptides and their mimics. Chem Biol Drug Des. 2012:80:787-809.

32. Amarzguioui M, Rossi JJ, Kim D. Approaches for chemically synthesized siRNA and vector-mediated RNAi. FEBS Lett. 2005;579:5974-81.

33. Braasch DA, Jensen S, Liu Y, Kaur K, Arar K, White MA, et al. RNA interference in mammalian cells by chemically-modified RNA. Biochemistry. 2003;42:7967-75.

34. Felgner PL, Ringold GM. Cationic liposome-mediated transfection. Nature. 1989;337:387-8.
35. Gary DJ, Puri N, Won YY. Polymer-based siRNA delivery: perspectives on the fundamental and phenomenological distinctions from polymer-based DNA delivery. J Control Release. 2007;121:64-73.

36. Malone RW, Felgner PL, Verma IM. Cationic liposome-mediated RNA transfection. Proc Natl Acad Sci U S A. 1989;86:6077-81.

37. Molitoris BA, Dagher PC, Sandoval RM, Campos SB, Ashush H, Fridman E, et al. siRNA targeted to p53 attenuates ischemic and cisplatin-induced acute kidney injury. J Am Soc Nephrol. 2009;20:1754-64.

38. Zheng $X$, Zhang $X$, Sun $H$, Feng B, Li M, Chen G, et al. Protection of renal ischemia injury using combination gene silencing of complement 3 and caspase 3 genes. Transplantation. 2006;82:1781-6.

39. Himmelfarb J, Joannidis M, Molitoris B, Schietz M, Okusa MD, Warnock D, et al. Evaluation and initial management of acute kidney injury. Clin J Am Soc Nephrol. 2008;3:962-7.

40. Zhang D, Liu Y, Wei Q, Huo Y, Liu K, Liu F, et al. Tubular p53 regulates multiple genes to mediate AKI. J Am Soc Nephrol. 2014;25:2278-89.

41. Rabb H, O'Meara YM, Maderna P, Coleman P, Brady HR. Leukocytes, cell adhesion molecules and ischemic acute renal failure. Kidney Int. 1997:51:1463-8.

42. Sheridan AM, Bonventre JV. Cell biology and molecular mechanisms of injury in ischemic acute renal failure. Curr Opin Nephrol Hypertens. 2000;9:427-34.

43. Vaseva AV, Moll UM. The mitochondrial p53 pathway. Biochim Biophys Acta. 2009;1787:414-20.

44. Tsapepas DS, Powell JT, Martin ST, Hardy MA, Ratner LE. An update to managing renal transplant ischemia reperfusion injury: novel therapies in the pipeline. Clin Transplant. 2013;27:647-8.

45. Powell JT, Tsapepas DS, Martin ST, Hardy MA, Ratner LE. Managing renal transplant ischemia reperfusion injury: novel therapies in the pipeline. Clin Transplant. 2013;27:484-91.

46. Fujino T, Muhib S, Sato N, Hasebe N. Silencing of p53 RNA through transarterial delivery ameliorates renal tubular injury and downregulates GSK-3beta expression after ischemia-reperfusion injury. Am J Physiol Renal Physiol. 2013;305:F1617-27.

47. Peng J, Li X, Zhang D, Chen JK, Su Y, Smith SB, et al. Hyperglycemia, p53, and mitochondrial pathway of apoptosis are involved in the susceptibility of diabetic models to ischemic acute kidney injury. Kidney Int. 2015;87:137-50.

48. Liu L, Li Y, Hu Z, Su J, Huo Y, Tan B, et al. Small interfering RNA targeting Toll-like receptor 9 protects mice against polymicrobial septic acute kidney injury. Nephron Exp Nephrol. 2012;122:51-61.

49. Shimizu H, Hori Y, Kaname S, Yamada K, Nishiyama N, Matsumoto S, et al. siRNA-based therapy ameliorates glomerulonephritis. J Am Soc Nephrol. 2010;21:622-33.

50. Motzer RJ, Bander NH, Nanus DM. Renal-cell carcinoma. N Engl J Med. 1996;335:865-75.

51. Ronkainen $\mathrm{H}$, Vaarala MH, Hirvikoski P, Ristimaki A. HuR expression is a marker of poor prognosis in renal cell carcinoma. Tumour Biol. 2011;32:481-7.

52. Danilin S, Sourbier C, Thomas L, Lindner V, Rothhut S, Dormoy V, et al. Role of the RNA-binding protein HuR in human renal cell carcinoma. Carcinogenesis. 2010;31:1018-26.

53. Shang D, Liu Y, Yang P, Chen Y, Tian Y. TGFBl-promoted adhesion, migration and invasion of human renal cell carcinoma depends on inactivation of von Hippel-Lindau tumor suppressor. Urology. 2012;79:966. e961-967.

54. Juengel E, Dauselt A, Makarevic J, Wiesner C, Tsaur I, Bartsch G, et al. Acetylation of histone $\mathrm{H} 3$ prevents resistance development caused by chronic mTOR inhibition in renal cell carcinoma cells. Cancer Lett. 2012:324:83-90.

55. Ge Q, Xu JJ, Evans DM, Mixson AJ, Yang HY, Lu PY. Leveraging therapeutic potential of multi-targeted siRNA inhibitors. Future Med Chem. 2009;1:1671-81.

56. Liu K, Chen H, You Q, Shi H, Wang Z. The siRNA cocktail targeting VEGF and HER2 inhibition on the proliferation and induced apoptosis of gastric cancer cell. Mol Cell Biochem. 2014;386:117-24.

57. Jackson AL, Bartz SR, Schelter J, Kobayashi SV, Burchard J, Mao M, et al. Expression profiling reveals off-target gene regulation by RNAi. Nat Biotechnol. 2003;21:635-7.

58. Jackson AL, Burchard J, Schelter J, Chau BN, Cleary M, Lim L, et al. Widespread siRNA "off-target" transcript silencing mediated by seed region sequence complementarity. RNA. 2006;12:1179-87.

59. Jackson AL, Linsley PS. Recognizing and avoiding siRNA off-target effects for target identification and therapeutic application. Nat Rev Drug Discov. 2010;9:57-67. 
60. Lin X, Ruan X, Anderson MG, McDowell JA, Kroeger PE, Fesik SW, et al. siRNA-mediated off-target gene silencing triggered by a $7 \mathrm{nt}$ complementation. Nucleic Acids Res. 2005;33:4527-35.

61. Kabilova TO, Meschaninova MI, Venyaminova AG, Nikolin VP, Zenkova MA, Vlassov W, et al. Short double-stranded RNA with immunostimulatory activity: sequence dependence. Nucleic Acid Ther. 2012;22:196-204.

62. Robbins $M$, Judge $A$, MacLachlan I. siRNA and innate immunity. Oligonucleotides. 2009;19:89-102.

63. Yang C, Li L, Xue Y, Zhao Z, Zhao T, Jia Y, et al. Innate immunity activation involved in unprotected porcine auto-transplant kidneys preserved by naked caspase-3 siRNA. J Transl Med. 2013;11:210.

64. Bell JK, Askins J, Hall PR, Davies DR, Segal DM. The dsRNA binding site of human Toll-like receptor 3. Proc Natl Acad Sci U S A. 2006;103:8792-7.

65. Choe J, Kelker MS, Wilson IA. Crystal structure of human toll-like receptor 3 (TLR3) ectodomain. Science. 2005;309:581-5.

66. Alexopoulou L, Holt AC, Medzhitov R, Flavell RA. Recognition of doublestranded RNA and activation of NF-kappaB by Toll-like receptor 3. Nature. 2001;413:732-8.

67. Hornung V, Guenthner-Biller M, Bourquin C, Ablasser A, Schlee M, Uematsu $S$, et al. Sequence-specific potent induction of IFN-alpha by short interfering RNA in plasmacytoid dendritic cells through TLR7. Nat Med. 2005;11:263-70.

68. Judge AD, Sood V, Shaw JR, Fang D, McClintock K, MacLachlan I. Sequence-dependent stimulation of the mammalian innate immune response by synthetic siRNA. Nat Biotechnol. 2005;23:457-62.

69. Sioud M. Induction of inflammatory cytokines and interferon responses by double-stranded and single-stranded siRNAs is sequence-dependent and requires endosomal localization. J Mol Biol. 2005;348:1079-90.

70. Racz Z, Godo M, Revesz C, Hamar P. Immune activation and target organ damage are consequences of hydrodynamic treatment but not delivery of naked siRNAs in mice. Nucleic Acid Ther. 2011;21:215-24.

71. Mueller TF, Reeve J, Jhangri GS, Mengel M, Jacaj Z, Cairo L, et al. The transcriptome of the implant biopsy identifies donor kidneys at increased risk of delayed graft function. Am J Transplant. 2008:8:78-85.

72. Wilflingseder J, Sunzenauer J, Toronyi E, Heinzel A, Kainz A, Mayer B, et al. Molecular Pathogenesis of Post-Transplant Acute Kidney Injury: Assessment of Whole-Genome mRNA and MiRNA Profiles. PLoS One. 2014;9:e104164.

73. Wu D, Zhu D, Xu M, Rong R, Tang Q, Wang X, et al. Analysis of transcriptional factors and regulation networks in patients with acute renal allograft rejection. J Proteome Res. 2011;10:175-81.

74. Wu D, Liu X, Liu C, Liu Z, Xu M, Rong R, et al. Network analysis reveals roles of inflammatory factors in different phenotypes of kidney transplant patients. J Theor Biol. 2014;362:62-8.

75. Grimm D, Streetz KL, Jopling CL, Storm TA, Pandey $K$, Davis $C R$, et al. Fatality in mice due to oversaturation of cellular microRNA/short hairpin RNA pathways. Nature. 2006;441:537-41.

76. Ojima I. Guided molecular missiles for tumor-targeting chemotherapy-case studies using the second-generation taxoids as warheads. Acc Chem Res. 2008:41:108-19.

77. Mokhtarieh AA, Cheong S, Kim S, Chung BH, Lee MK. Asymmetric liposome particles with highly efficient encapsulation of siRNA and without nonspecific cell penetration suitable for target-specific delivery. Biochim Biophys Acta. 1818;2012:1633-41.

78. Valencia-Serna J, Gul-Uludag H, Mahdipoor P, Jiang X, Uludag H. Investigating siRNA delivery to chronic myeloid leukemia K562 cells with lipophilic polymers for therapeutic BCR-ABL down-regulation. J Control Release. 2013;172:495-503.

79. Oe Y, Christie RJ, Naito M, Low SA, Fukushima S, Toh K, et al. Actively-targeted polyion complex micelles stabilized by cholesterol and disulfide cross-linking for systemic delivery of siRNA to solid tumors. Biomaterials. 2014;35:7887-95.

80. Zhou J, Neff CP, Liu X, Zhang J, Li H, Smith DD, et al. Systemic administration of combinatorial dsiRNAs via nanoparticles efficiently suppresses HIV-1 infection in humanized mice. Mol Ther. 2011;19:2228-38.

\section{Submit your next manuscript to BioMed Central and take full advantage of:}

- Convenient online submission

- Thorough peer review

- No space constraints or color figure charges

- Immediate publication on acceptance

- Inclusion in PubMed, CAS, Scopus and Google Scholar

- Research which is freely available for redistribution

Submit your manuscript at www.biomedcentral.com/submit 